\title{
Macroinvertebrados acuáticos como indicadores de calidad hídrica en áreas de descargas residuales al río Quevedo, Ecuador
}

\author{
Aquatic macroinvertebrates as indicators of water quality in areas of residual discharge to the \\ Quevedo river, Ecuador
}

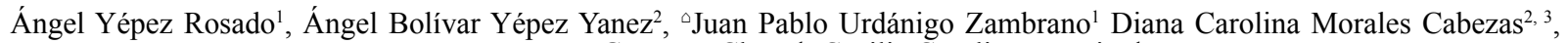
Norma María Guerrero Chuez ${ }^{1}$, Cecilia Carolina TayHing ${ }^{1}$

${ }^{1}$ Universidad Técnica Estatal de Quevedo. Facultad de Ciencias Ambientales, Carrera de Ingeniería en Gestión Ambiental. Campus Manuel Haz Álvarez. Av. Quito Km 1.5 vía Santo Domingo de los Tsáchilas. EC.120301. Quevedo, Ecuador. ayepez@uteq.edu.ec; ‘jurdanigo@uteq.edu.ec; nguerrero@uteq.edu.ec; ctayhing@uteq.edu.ec

${ }^{2}$ Universidad Técnica Estatal de Quevedo. Facultad de Ciencias Empresariales, Carrera de Ingeniería en Gestión Empresarial. Campus Manuel Haz Álvarez. Av. Quito Km 1.5 vía Santo Domingo de los Tsáchilas. EC.120301. Quevedo, Ecuador. byepez@uteq.edu.ec; diana.morales@uteq.edu.ec

${ }^{3}$ Unidad Educativa Liceo Héroes del 41, Departamento de Talento Humano. km 2 vía Valencia. Quevedo, Ecuador

Rec.: 26.09.2016. Acept.: 16.01.2017.

Publicado el 1 de junio de 2017

\section{Resumen}

E trabajo tuvo como objetivos caracterizar las condiciones físico-químicas del agua, identificar la diversidad de macroinvertebrados bentónicos y determinar la calidad hídrica en dos sitios urbanos de monitoreo del río en la ciudad de Quevedo. Los muestreos se realizaron desde septiembre a noviembre del 2015, en sitios influenciados por descargas de efluentes residenciales (ER) y agrícolas-industriales (EAI). Se midieron los parámetros físico-químicos de calidad del agua: oxígeno disuelto, demanda bioquímica de oxígeno, demanda química de oxígeno y sólidos disueltos totales. Se estimaron los índices de riqueza CHAO2, diversidad de ShannonWeiner (H), dominancia de Simpson (1-D) y disimilitud de Bray-Curtis. La calidad del agua fue estimada con el índice Biological Monitoring Working Party (BMWP-Col). Los valores de los parámetros físico-químicos no presentaron diferencias entre ER y EAI. No existieron diferencias estadísticas entre la riqueza de familias esperada (CHAO2) y la riqueza observada. La familia Tubificidae mostró la mayor abundancia total con 4574 individuos (90.48\%), para ER 3918 individuos (93\%) y EAI $656(76 \%)$. La diversidad $\mathrm{H}$ en ER y EAI fue baja $0.49 \pm 0.22 ; 1.009 \pm 0.21$ respectivamente, y difirieron significativamente. La dominancia más alta la presentó ER $(0.78 \pm 0.1)$, y difirió de EAI (0.58 \pm 0.096$)$. El índice BMWP-Col indicó que ER registró calidad de agua

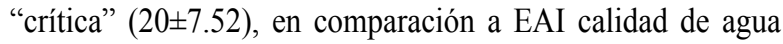
"dudosa" (37 \pm 6.27$)$. El cuerpo hídrico que atraviesa la zona urbana de Quevedo se encontró fuertemente contaminado a causa del urbanismo y las actividades agrícolas e industriales lo que influye negativamente en la estructura de las comunidades de macroinvertebrados acuáticos.

Palabras clave: Calidad del agua, río urbano, BMWP-Col, Tubificidae, bioindicadores.
$\mathrm{T}_{\mathrm{a}}^{\mathrm{h}}$ he objective of this paper was to characterize physical and chemical conditions of water, to identify the diversity of benthic macroinvertebrates, and to determine water quality in two urban monitoring sites of the river in the city of Quevedo. Samplings were carried out from September to November 2015, at sites influenced by residential (RE) and agricultural-industrial (EAI) discharges. Physio-chemical parameters of water quality were measured: dissolved oxygen, biochemical oxygen demand, chemical oxygen demand and total dissolved solids. CHAO2 richness indexes, Shannon-Weiner diversity (H), Simpson dominance (1-D) and BrayCurtis dissimilarity were estimated. Water quality was estimated using the Biological Monitoring Working Party (BMWP-Col) index. The values of the physical-chemical parameters did not show differences between RE and IAS. There were no statistical differences between the expected family richness (CHAO2) and the observed richness. The Tubificidae family showed the highest total abundance with 4574 individuals (90.48\%), for ER - 3918 individuals (93\%), and EAI - 656 (76\%). H-diversity in ER and IAC was low: $0.49 \pm 0.22 ; 1.009 \pm 0.21$ respectively, and differed significantly. The highest dominance was shown by ER $(0.78 \pm 0.1)$, and it differed from IAC (0.58 \pm 0.096$)$. The BMWP-Col index indicated that ER recorded "critical" water quality (20 \pm 7.52$)$, as compared to "uncertain" water quality $(37 \pm 6.27)$. The water body that crosses the urban area of Quevedo was found heavily polluted due to urbanism, agricultural and industrial activities, negatively influencing the structure of aquatic macroinvertebrate communities.

Key words: Water quality, urban river, BMWP-Col, Tubificidae, bioindicators. 


\section{Introducción}

$\mathrm{L}$ as actividades humanas como la agricultura, la expansión residencial, el desarrollo de embalses así como las alteraciones hidrológicas de los cuerpos de agua pueden cambiar las condiciones ambientales del agua y afectar así la presencia de macroinvertebrados acuáticos (Damanik-Ambarita et al., 2016). La contaminación de las cuencas hídricas produce pérdida de biodiversidad teniendo implicaciones, como disminución de la resiliencia, simplificación del sistema y pérdida de integridad ecológica (Gualdoni \& Oberto, 2012). En Ecuador, el río Quevedo es contaminado por actividades relacionadas con los asentamientos humanos (contaminación por el vertido de aguas grises o negras, vertido de efluentes de desechos sólidos a cielo abierto), agricultura (vertido de productos químicos) e industria (vertido de efluentes y lubricantes), provocando pérdida de la biodiversidad y disminución de la calidad ambiental del recurso hídrico (Gobierno autónomo descentralizado municipal del cantón Quevedo, 2016).

En cuanto al monitoreo de la calidad del agua, las variables físico-químicas solo dan una idea puntual sobre la calidad del agua; no obstante, el monitoreo biológico informa sobre las variaciones en el tiempo (Alba-Tercedor, 1996; Roldán, 2003), conociendo que un organismo es buen indicador de calidad del agua cuando se encuentra invariablemente en un ecosistema de características definidas y cuando su población es superior al resto de los organismos con los que comparte el mismo hábitat (Lozano, 2005). En este contexto, el creciente interés por conocer y proteger los ecosistemas fluviales y estudiar sus cambios en el tiempo, ha estimulado en las últimas décadas el desarrollo de criterios biológicos que permitan estimar el efecto de las intervenciones humanas en ellos (Figueroa et al., 2003). Estudios de diversidad de macroinvertebrados acuáticos y de su papel en la determinación de calidad del agua en el trópico, son numerosos; Posada-García \& Roldan-Pérez (2003); Chará et al. (2010); Meza-S et al. (2012); AriasHidalgo et al. (2013); Giraldo et al. (2014). En Ecuador, Alvarez-Mieles et al. (2013) evaluaron el estado y el manejo ecológico del Humedal Abras de Mantequilla mediante el empleo de índices bióticos y su relación con factores abióticos. Damanik-Ambarita et al. (2016) evaluaron la calidad del agua en la cuenca del río Guayas en sitios influenciados por diferentes usos de suelo, las muestras tomadas en ríos con poca intervención humana presentaron una buena calidad del agua en comparación con áreas residenciales que presentaron una mala o muy mala calidad del agua. Ríos-Touma et al. (2011) determinaron que la composición de las comunidades de macroinvertebrados difiere entre estaciones, existiendo un fuerte incremento de la riqueza, densidad y diversidad de macroinvertebrados acuáticos en la estación seca. No obstante, a pesar de estos esfuerzos, no existen estudios formales que evalúen los impactos de las actividades de los asentamientos humanos (urbanismo, agricultura e industria) en la integridad ecológica y calidad del agua en el río Quevedo.

Teniendo en cuenta esta situación y el creciente uso de los indicadores biológicos como complemento de las investigaciones de calidad del agua, el presente trabajo tuvo como objetivos: (1) caracterizar las condiciones físico-químicas del agua, (2) identificar la diversidad de macroinvertebrados bentónicos y (3) determinar la calidad hídrica con un índice basado en el ensamblaje de macroinvertebrados de dos sitios de muestreo del río Quevedo ubicadas en la zona urbana de la ciudad de Quevedo. Se espera encontrar en la zona urbana del río Quevedo familias de macroinvertebrados bentónicos indicadoras de mala calidad del agua. Los resultados de este estudio podrían ayudar a los interesados en el manejo de la cuenca hídrica del río Quevedo (autoridades locales, comunidad en general), con el aporte de las bases para desarrollar actividades de gestión ambiental y de vigilancia encaminados a la recuperación de la calidad hídrica del río Quevedo.

\section{Materiales y métodos}

E l área de estudio se localizó en la subcuenca alta del río Vínces, a unos 80 msnm, en el cantón Quevedo, provincia Los Ríos, Ecuador. La recolección de los macroinvertebrados acuáticos se llevó a cabo durante los meses de septiembre a noviembre (época seca) del 2015. Se muestrearon dos sitios en la zona urbana del río Quevedo, el primero influenciado con descargas de efluentes residenciales de corriente rápida, sustrato lodoso-arenoso, poca hojarasca y una sección pequeña de grandes árboles (ER X: 670760.43 Y: 9884920.49); el segundo con descargas de efluentes de plantaciones agrícolas-industriales, corriente lenta, sustrato lodosoarenoso y poca hojarasca (EAI; X: 672378.28 Y: 9889042.90).

\section{Trabajo de campo y laboratorio}

Se efectuaron cuatro muestreos periódicos cada 20 días, fueron medidos in situ los parámetros físicoquímicos de calidad del agua: oxígeno disuelto (OD), demanda bioquímica de oxígeno (DBO), demanda química de oxígeno (DQO) y sólidos disueltos totales (SDT) mediante un medidor multiparamétrico (Hanna HI 9829). Con una Red D-net por sitio de muestreo, se ejecutaron arrastres removiendo hojarascas y 
sedimento in situ en el cauce en una extensión de 100 $\mathrm{m}$ longitudinales en sentido contrario de la corriente (Fernández y Domínguez, 2001; Roldán, 2003). Las muestras recogidas fueron etiquetadas y almacenadas en recipientes con alcohol al $70 \%$ en el laboratorio de Biotecnología de la Universidad Técnica Estatal de Quevedo. La identificación taxonómica de los especímenes llegó hasta nivel de familia utilizando un estereoscopio y claves taxonómicas especializadas (Posada-García y Roldan, 2003; Domínguez et al., 2006).

\section{Análisis de datos}

Se realizaron pruebas de normalidad de ShapiroWilk a los datos obtenidos de los parámetros físicoquímicos, índices de diversidad, índice de calidad hídrica BMWP-Col, para comprobar el cumplimiento de las asunciones de las pruebas paramétricas. Se evaluaron las características de la estructura de la comunidad de macroinvertebrados: abundancia, riqueza, diversidad y dominancia. Se calculó la diversidad mediante el índice de Shannon-Wiener $(\mathrm{H})$, la dominancia a través del índice de Simpson (1-D), el número total esperado de taxones con el método de estimación de riqueza Chao 2 con Bootstrap de 1000 interacciones; además, se comparó la composición de los sitios de muestreo con el índice de disimilitud Bray-Curtis.

Las variables físico-químicas de los sitios de muestreo del río Quevedo fueron comparadas entre sí mediante la prueba t-Student. Para estimar el efecto de las descargas de efluentes en la zona urbana del río Quevedo sobre la diversidad de macroinvertebrados acuáticos, se aplicó una prueba t-Student a los índices $\mathrm{H}$ y 1-D entre ER y EAI. La comparación de la riqueza de familias observada con la esperada (CHAO2) se realizó con una prueba de bondad de ajuste (Ludwig y Reynolds, 1988). La comparación de la composición de familias de macroinvertebrados acuáticos entre los sitios se realizó mediante un dendrograma (aglomeración por ligamiento promedio bajo el algoritmo UPGMA) a partir de la matriz de disimilitud mediante el índice de BrayCurtis (Ludwig y Reynolds, 1988; Hammer et al. 2001; Moreno, 2001).
Para el análisis de la calidad hídrica se utilizó el Índice BMWP-Col, de acuerdo a la escala adaptada para Colombia por Roldán (2003). Para determinar si existían diferencias estadísticas en la calidad del agua entre los sitios de muestreo (zona residencial y zona agrícola industrial) de la zona urbana del río Quevedo se empleó una prueba t-Student entre las puntuaciones de cada salida del índice de Calidad BMWP-Col. Todos los análisis se realizaron con un nivel de significancia $\mathrm{p}=0.05$ en el programa Past versión 3 (Hammer et al., 2001).

\section{Resultados y discusión}

\section{Variables físico-químicas}

Los valores promedios de los parámetros de calidad de agua son similares entre los sitios de muestreo (Cuadro 1). Estos valores son similares a los reportados por Valverde et al. (2009) en estaciones de muestreo influenciadas por descargas de zonas urbanas del municipio de Envigado en la quebrada La Ayurá, Colombia. El OD y SDT en el estudio difieren de lo reportado por Montoya (2008) quien obtuvo en dos estaciones de muestreo, ubicadas en la zona urbana de la microcuenca Cimarronas, Colombia, valores de OD entre 5.02-6.11 $\mathrm{mgL}^{-1}$ y SDT de 13-191 $\mathrm{mgL}^{-1}$. Figueroa et al. (2003) registran valores promedios similares para OD (6.61 \pm 1.51$)$, DQO (12.84 \pm 3.17$)$ y DBO (2.31 \pm 1.96$)$, para ríos del sur de Chile con usos de suelo agrícolaganadero, forestal y urbano-industrial.

Los valores de oxígeno disuelto obtenidos en el estudio indican niveles relativamente buenos de oxígeno, este gas depende de las características del cauce, la turbulencia del agua y los procesos químicos y biológicos e influye en la riqueza y los patrones de distribución de las familias de macroinvertebrados (Guerrero-Bolaño et al., 2003). Los sólidos disueltos totales variaron, se consideran dentro de los valores típicos para aguas continentales neotropicales (Roldán y Ramírez, 2008). La demanda química del oxígeno en los sitios de muestreo señalaron un grado de contaminación

Cuadro 1. Estadísticos descriptivos de parámetros físico-químicos de calidad de agua en dos sitios de muestreo del río Quevedo, Ecuador

\begin{tabular}{lrrrrr}
\hline Parámetro $\left(\mathbf{m g L}^{-1}\right)$ & \multicolumn{1}{c}{ ER } & \multicolumn{1}{c}{ EAI } & \multicolumn{1}{c}{ Promedio } & (t) & \multicolumn{1}{c}{ (p) } \\
\hline Oxígeno disuelto & $7.26 \pm 0.41$ & $6.94 \pm 0.57$ & $7.09 \pm 0.49$ & 0.903 & 0.401 \\
Demanda biológica de oxígeno & $1.26 \pm 0.32$ & $0.77 \pm 0.67$ & $1.01 \pm 0.55$ & 1.316 & 0.235 \\
Demanda química de oxígeno & $15.25 \pm 3.96$ & $19.33 \pm 3.62$ & $17.28 \pm 4.13$ & -1.521 & 0.179 \\
Sólidos disueltos totales & $14.80 \pm 0.94$ & $14.28 \pm 2.11$ & $14.53 \pm 1.53$ & 0.454 & 0.665 \\
\hline
\end{tabular}

ER: efluentes residenciales. EAI: efluentes agrícolas-industriales 
bajo; es decir no se requiere de gran cantidad de oxígeno para la oxidación tanto de materia orgánica como inorgánica (Patiño, 2015). Diferentes autores resaltan que los factores fisicoquímicos del medio acuático, como $\mathrm{pH}$, conductividad, oxígeno disuelto $\mathrm{y}$ temperatura, son determinantes en la distribución de los macroinvertebrados acuáticos, donde los organismos son más sensibles (Morelli y Verdi, 2014).

\section{Diversidad de macroinvertebrados}

En el cuerpo hídrico analizado se recolectaron un total de 5055 macroinvertebrados acuáticos, 4193 individuos en ER y 862 individuos en EAI, pertenecientes a 5 phylum, 8 clases, 9 órdenes y 14 familias. No existieron diferencias significativas entre la riqueza de familias observadas con el índice $\mathrm{CHAO} 2$ (riqueza esperada) en ER y EAI ( $p=0.406 ; p=0.119$; respectivamente). La familia Tubificidae mostró la mayor abundancia en ER con 3918 individuos (93\%), seguidas por Glossiphonidae con 149 individuos (4\%) y Lymnaeidae 115 (2\%). En EAI la familia Tubificidae fue la más abundante con 656 individuos (76\%), seguidos por Baetidae con 41 individuos (5\%) y Lymnaeidae 35 (4\%) (Cuadro 2). Resultados similares fueron registrados por Rivera et al. (2013) en el humedal urbano Andino de Colombia, donde la familia Tubificidae fue la más abundante en las estaciones de monitoreo influenciadas con descargas urbanas. Valverde et al. (2009) registraron abundancias similares en la familia Tubificidae en estaciones de muestreo influenciadas por descargas de zonas urbanas del municipio de Envigado en la quebrada La Ayurá, Colombia. La familia Tubificidae es característica de sistemas eutrofizados de abundante materia orgánica en descomposición, presentes en zonas extremas de contaminación (Roldán, 2003).

La mayor diversidad H la mostró EAI (1.009 \pm 0.21$)$; mientras que ER mostró la menor diversidad $\mathrm{H}$ con $0.49 \pm 0.22$, existiendo diferencias en las puntuaciones de diversidad H entre ER y EAI $(\mathrm{p}<0.0001)$ (Cuadro 2). Resultados similares fueron reportados en la quebrada La Cascosa, Huila, Colombia, afectada por vertimientos de origen urbanos, donde se registraron valores de diversidad H entre 0.5-1.0 (Portilla, 2015). Estos resultados difieren con lo registrado por Valverde et al. (2009) en donde la diversidad Shannon-Wienner es superior $(2.28 \pm 0.34)$ a lo registrado en este estudio en aguas con influencia de descargas residuales urbanas. Así mismo, resultados distintos de diversidad $\mathrm{H}$ también presentaron Correa-Araneda et al. (2010) en estaciones de monitoreo de zonas urbanas en ríos del sur de Chile, en donde la diversidad se mantuvo en rangos de 1.501.95 .

La diversidad de macroinvertebrados bentónicos en los ríos urbanos se encuentran influenciados por las fuentes de contaminación puntuales y difusas asociados a la urbanización, actividades agrícolas e industriales, lo que simplifica su estructura, repercutiendo en menor riqueza y diversidad de familias (Pavé y Marchese, 2005; Portilla, 2015). En consecuencia, la diversidad de macroinvertebrados acuáticos registrada en el estudio presentó valores menores a uno, rangos entre uno a tres, son característicos de medios acuáticos moderadamente contaminados (Wilhm y Dorris, 1968).

Las puntuaciones del índice de dominancia de Simpson (1-D) indicaron que la mayor dominancia se encontró en ER $(0.78 \pm 0.1)$, mientras, EAI presentó la menor dominancia $(0.58 \pm 0.096)$ con diferencias significativas entre ER y EAI $(p<0.0001)$ (Cuadro 2). Portilla (2015) registraron valores de dominancia entre $0.17-0.62$, de mayor dominancia en estaciones de monitoreo correspondientes a zonas urbanas. Las diferencias en la dominancia entre sitios de muestreo podrían estar influenciadas por las condiciones del ambiente acuático; cuando la comunidad de macroinvertebrados es afectada con un descenso de la diversidad de insectos acuáticos sensibles a causa de la abundante materia orgánica en descomposición, aumentando la dominancia de familias tolerantes con fuentes adicionales de alimento (Metcalfe, 1989; Herbas et al., 2006).

El índice de disimilitud Bray-Curtis indicó que existe una composición diferente entre familias en los puntos de muestreo (30\%) (Figura 1), probablemente las diferencias estarían influenciadas por la contaminación del medio en ER, lo que favorece la llegada de descargas orgánicas o químicas al lecho del sitio de muestreo, afectando la comunidad acuática allí establecida (Posada et al., 2000).

\section{Calidad del agua}

El índice de calidad del agua BMWP-Col indicó que el agua en ER fue de calidad crítica (aguas muy contaminadas) (20.0 \pm 7.52$)$, mientras, en EAI el agua fue de calidad dudoso (aguas moderadamente contaminadas) (37 \pm 6.27$)$ (Cuadro 2), existieron diferencias significativas en la calidad del agua entre los dos sitios de muestreo $(\mathrm{p}=0.0133)$. Las diferencias entre ER y EAI se deben principalmente a la presencia de las familias Perlidae y Baetidae en el sitio de muestreo con descargas de efluentes agrícola-industrial (EAI), no registradas en ER, aunque estas familias son indicadoras de poca tolerancia a la contaminación, la presencia de estas podrían estar relacionadas por otros factores además de la calidad del agua, como la velocidad de la corriente, la conducta de deriva en aguas en movimiento, que puede arrastrar algunas especies dentro de áreas (río-abajo), normalmente en ellos no ocurren (Rosenberg y Resh, 1993). Valores 


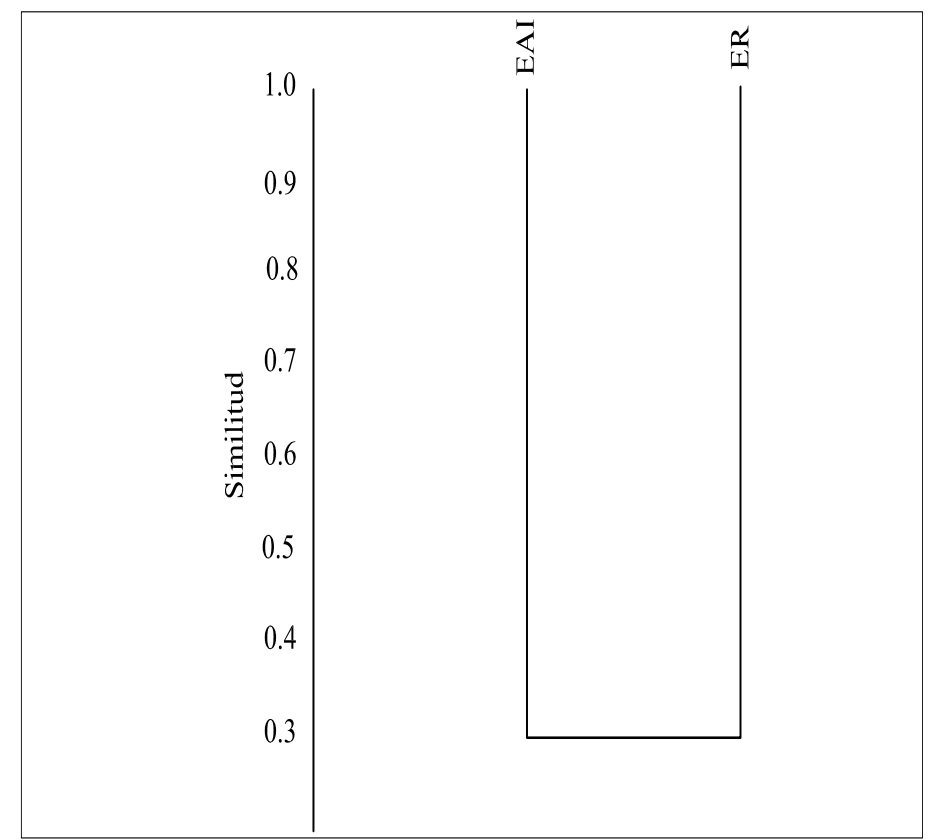

Figura 1. Análisis de agrupamiento entre los sitios de efluente agrícolas-industriales (EAI) y efluentes residenciales (ER) con base en el índice disimilitud Bray-Curtis, método de distancia UPGMA

Cuadro 2. Abundancia de los taxones registrados en dos sitios de monitoreo en la zona urbana del río Quevedo, Ecuador

\begin{tabular}{|c|c|c|c|c|c|c|c|c|c|c|c|}
\hline \multirow{2}{*}{ Orden } & \multirow{2}{*}{ Familia } & \multicolumn{5}{|c|}{ ER } & \multicolumn{5}{|c|}{ EAI } \\
\hline & & 1 & 2 & 3 & 4 & Tot. & 1 & 2 & 3 & & Tot. \\
\hline Anisóptera & Libellulidae & 0 & 0 & 0 & 0 & 0 & 0 & 4 & 0 & 0 & 4 \\
\hline Amphipoda & Hyalellidae & 3 & 3 & 4 & 2 & 12 & 1 & 1 & 9 & 1 & 12 \\
\hline Coleoptera & Elmidae & 2 & 0 & 1 & 2 & 5 & 0 & 0 & 4 & 2 & 6 \\
\hline Coleoptera & Psephenidae & 0 & 0 & 0 & 0 & 0 & 1 & 2 & 0 & 1 & 4 \\
\hline Diptera & Ceratopogonidae & 1 & 2 & 2 & 7 & 12 & 8 & 10 & 1 & 2 & 21 \\
\hline Diptera & Chironomidae & 3 & 1 & 2 & 3 & 9 & 0 & 5 & 0 & 2 & 7 \\
\hline Diptera & Simulidae & 2 & 0 & 0 & 0 & 2 & 0 & 5 & 0 & 3 & 8 \\
\hline Diptera & Tipulidae & 0 & 0 & 0 & 4 & 4 & 0 & 1 & 0 & 2 & 3 \\
\hline Ephemeroptera & Baetidae & 0 & 0 & 0 & 0 & 0 & 23 & 15 & 0 & 3 & 41 \\
\hline Haplotaxida & Tubificidae & 3138 & 240 & 297 & 243 & 3918 & 228 & 100 & 200 & 128 & 656 \\
\hline Plecoptera & Perlidae & 0 & 0 & 0 & 0 & 0 & 11 & 6 & 10 & 8 & 35 \\
\hline Pulmonata & Lymnaeidae & 35 & 0 & 12 & 28 & 75 & 21 & 0 & 12 & 2 & 35 \\
\hline Rynchobdellida & Glossiphonidae & 88 & 25 & 36 & 0 & 149 & 11 & 2 & 10 & 4 & 27 \\
\hline Seriata & Planariidae & 1 & 0 & 4 & 2 & 7 & 1 & 0 & 2 & 0 & 3 \\
\hline \multicolumn{12}{|l|}{ Índices } \\
\hline BMWP & & \multicolumn{5}{|c|}{26.009 .0022 .0023 .00} & \multicolumn{5}{|c|}{36.0044 .0029 .0039 .00} \\
\hline \multicolumn{2}{|c|}{ Diversidad Shannon } & 0.21 & 0.43 & 0.67 & 0.67 & & 0.98 & 1.31 & 0.82 & 0.90 & \\
\hline \multicolumn{2}{|c|}{ Dominancia Simpson (1-D) } & 0.07( & 0.20 & 0.30 & 0.29 & & 0.42 & 0.54 & 0.34 & 0.33 & \\
\hline
\end{tabular}

Sitio de muestreo influenciado con efluentes residenciales (ER); sitio de muestreo influenciado con efluentes agrícolas-industriales (EAI). 
similares de calidad de agua se registró en la quebrada La Marinilla influenciada por uso de suelo agrícola industrial, de la cuenca del río Negro, Colombia, con una calidad "Dudosa" (Forero et al., 2014). Asi mismo, el índice BMWP-Col tuvo calificación "Muy crítico" por estaciones de muestreo influenciados con efluentes de aguas residuales en el Río Frío, Colombia, que evidencia el impacto del crecimiento urbano sobre los cuerpos de agua que circulan y fluyen en las ciudades (Restrepo, 2013). De la misma manera, DamanikAmbarita et al. (2016) obtuvieron valores de calidad de agua "críticos" a "muy críticos" en sitios de monitoreo cercanos a áreas urbanizadas. Portilla (2015) identificó en estaciones de muestreo (zona urbana) de la quebrada Cascajosa, valores "Aceptables" de calidad de agua. Las diferencias entre las puntuaciones del índice BMWP-Col estarían relacionadas a la pendiente del canal y al tipo de sustrato (rocas grandes) de la quebrada Cascajosa, que influyen en la turbulencia del agua y por consiguiente en el incremento de la concentración de oxígeno, factor importante para la recuperación de la calidad del agua, debido a que incrementa la capacidad oxidante de la quebrada para mineralizar los aportes de materia orgánica.

\section{Conclusiones}

Se evidenció que los sitios de muestreo del cuerpo Shídrico que atraviesa la zona urbana de la ciudad de Quevedo se encuentran fuertemente contaminadas a causa de las actividades antropogénicas que se desarrollan en ella. La menor calidad hídrica la presentó el sitio de muestreo con influencia de descargas residenciales (ER), esto quedó evidenciado con las bajas puntuaciones de diversidad y del índice de calidad hídrica BMWP-Col (crítica). En este contexto, las actividades antrópicas influyeron negativamente en la estructura de las comunidades de insectos acuáticos, repercutiendo negativamente en el equilibrio ecológico del cuerpo de agua.

\section{Agradecimiento}

A la Universidad Técnica Estatal de Quevedo, Facultad de Ciencias Ambientales, Carrera de Ingeniería en Gestión Ambiental, por colaborar en el desarrollo del estudio.

\section{Bibliografía}

Alba-Tercedor, J. (1996). Macroinvertebrados acuaticos y calidad de las aguas de los ríos. 2: 203-213. Madrid, España: Instituto Tecnológico Geominero de España.

Alvarez-Mieles, G., Irvine, K., Griensven, AV., AriasHidalgo, M., Torres, A., \& Mynett, AE. (2013). Relationships between aquatic biotic communities and water quality in a tropical river-wetland system (Ecuador). Environmental Science and Policy, 34(december), 115-127. http://doi.org/10.1016/j. envsci.2013.01.011

Arias-Hidalgo, M., Bhattacharya, B., Mynett, A., \& Van Griensven, A. (2013). Experiences in using the TRMM data to complement raingauge data in the Ecuadorian coastal foothills. Journal of Hydrology and Earth System Sciences, 17 (9), 2905-2915.

Chará-Serna, AM., Chará, JD., Zúñiga, MC., Pedraza, GX. \& Giraldo, LP. (2010). Clasificación trófica de insectos acuáticos en ocho quebradas protegidas de la ecorregión cafetera colombiana. Universitas Scientiarum 15(1), 27-36.

Correa-Araneda, F., Rivera, R., Urrutia, J., \& R, P. D. L. (2010). Efectos de una zona urbana sobre la comunidad de macroinvertebrados bentónicos de un ecosistema fluvial del sur de Chile. Limnetica, 29(2), 183-194.

Damanik-Ambarita, MN., Lock, K., Boets, P., Everaert, G., Nguyen, THT., Forio, MAE., Goethals, PLM. (2016). Ecological water quality analysis of the Guayas river basin (Ecuador) based on macroinvertebrates indices. Limnologica - Ecology and Management of Inland Waters, 57, 27-59. http://doi.org/10.1016/j.limno.2016.01.001

Domínguez, E., Molineri, C., Pescador, ML., Hubbard, MD., \& Nieto, C. (2006). Ephemeroptera of South America. In Aquatic Biodiversity in Latin America Biodiversidad Acuatica en America Latina. 2, 646.

Fernández, HR., \& Domínguez, E. (2001). Guía para la determinación de los artrópodos bentónicos sudamericanos. BOOK, Universidad Nacional de Tucumán, Facultad de Ciencias Naturales e Instituto M. Lillo. Retrieved from https://books.google.com. ec/books?id=ZHMWAQAAIAAJ

Figueroa, R., Valdovinos, C., Araya, E., \& Parra, O. (2003). Macroinvertebrados bentónicos como indicadores de calidad de agua de ríos del sur de Chile. Revista Chilena de Historia Natural. JOUR, scielocl. Retrieved from http://www.scielo.cl/pdf/ rchnat/v76n2/art12.pdf

Forero, L., Longo, M., Ramírez, J., \& Chalar, G. (2014). Índice de calidad ecológica con base en 
macroinvertebrados acuáticos para la cuenca del río Negro ( ICE RN-MAE ), Colombia. Revista de Biologia Tropical, 62(2), 233-247.

Giraldo, A., Garcés-Restrepo, MF., Quintero-Angel, A., Bolívar, W. \& Velandia-Perilla, JH. (2014). Vertebrados Terrestres de Isla Palma (Bahía Málaga, Valle del Cauca). Bol.Cient. Mus. Hist. Nat. 18 (2)

Gobierno Autónomo Descentralizado Municipal del cantón Quevedo. (2016). Plan de desarrollo y ordenamiento territorial cantón Quevedo 2012 - 2016 (Segunda). Quevedo,Ecuador. Retrieved from http://app.sni.gob.ec/sni-link/sni/PORTAL SNI/data_sigad_plus/sigadplusdiagnostico/ PDyOT- actualizado 2014 - definitivo SENPLADES_14-11-2014.pdf

Gualdoni, CM., \& Oberto, AM. (2012). Estructura de la comunidad de macroinvertebrados del arroyo Achiras (Córdoba, Argentina): análisis previo a la construcción de una presa . Iheringia. Série Zoologia . JOUR, Scielo .

Guerrero-Bolaño, F., Manjarrés-Hernandéz, A., \& Núñez-Padilla, N. (2003). The Benthonic Macroinvertebrates of Pozo Azul (Gaira River Basin, Colombia) and their Relationship with Water Quality. Acta Biológica Colombiana, 8(2), 43-55.

Hammer, Ø., Harper, DAT. a. T., \& Ryan, PD. (2001). PAST: Paleontological Statistics Software Package for Education and Data Analysis. Palaeontologia Electrónica, 4(1)(1), 1-9. http://doi.org/10.1016/j. bcp.2008.05.025

Herbas, R., Rivero, F., Gonzales, R., \& Arce, O. (2006). Indicadores Biologicos de calidad del Agua. Universidad Mayor de San Simón. Universidad Mayor de San Simón. Retrieved from http://www. pnuma.org/agua-miaac/Curso Regional MIAAC/ Conferencias/Dia5(14-agosto-2010)/MIAAC PNUMAPANAGO10MAX/BIBLIOGRAFIA/ indicadoresBiologicosCalidadAgua.pdf

Lozano, L. (2005). La bioindicación de la calidad del agua: importancia de los macroinvertebrados en la cuenca alta del río Juan Amarillo, cerros orientales de Bogota. Umbral Científico, (7), 5-11. JOUR.

Ludwig, J., \& Reynolds, J. (1988). Statistical ecology. A primer on methods and computing. Ecological Modelling. Nueva York: John Wiley \& Sons. Retrieved from http://www2.ib.unicamp.br/profs/ thomas/NE002_2011/maio12/LR 1988 StatEcol sel1.pdf

Metcalfe, JL. (1989). Biological water quality assessment of running waters based on macroinvertebrate communities: History and present status in Europe. Environmental Pollution, 60(1-2), 101-139. http:// doi.org/10.1016/0269-7491(89)90223-6

Meza-S, AM., Días-G, L., Walteros-M, J. (2012). Calidad de agua y composición de macroinvertebrados acuáticos en la subcuenca alta del río Chinchiná. Caldasia 34(2), 443-456.

Montoya, Y. (2008). Caracterización de la biodiversidad acuática y de la calidad de las aguas de la quebrada Los Andes, El Carmen de Viboral, Antioquia. Revista Institucional Universidad Tecnológica del Chocó: Investigación, Biodiversidad y Desarrollo, 27(1), 85-91.

Morelli, E., \& Verdi, A. (2014). Diversidad de macroinvertebrados acuáticos en cursos de agua dulce con vegetación ribereña nativa de Uruguay. Revista Mexicana de Biodiversidad, 85(4), 11601170. http://doi.org/10.7550/rmb.45419

Moreno, CE. (2001). Métodos para medir la biodiversidad. M\&T Manuales y Tesis Sociedad Entomológica Aragonesa, 1(1), 84.

Patiño, G. (2015). Evaluación de la calidad del agua por medio de bioindicacores macroinvertebrados acuáticos en la quebrada La Vieja. Universidad Distrital Francisco José de Caldas.

Pavé, PJ., \& Marchese, M. (2005). Invertebrados bentónicos como indicadores de calidad del agua en ríos urbanos (Paraná-Entre Ríos, Argentina). Ecología Austral, 15: 183-197.

Portilla, N. (2015). Distribución espacial y temporal de macroinvertebrados acuáticos en la quebrada $\mathrm{La}$ Cascajosa - Garzón ( Huila ). ENTORNOS, 28(1), $56-75$.

Posada, JA., Roldán, G., \& Ramírez, JJ. (2000). Caracterización fisicoquímica y biológica de la calidad de aguas de la cuenca de la quebrada Piedras Blancas, Antioquia, Colombia. Revista de Biología Tropical, 48(1), 17.

Posada-García, J.; Roldan-Pérez, G. (2003). Clave Ilustrada Y Diversidad De Las Larvas De Trichoptera En El Nor-Occidente De Colombia. Caldasia, 25(1), 169-192.

Restrepo, M. (2013). Aplicación de índices físicoquímicosy biológicos para la determinación de la calidad del agua del río Frio. AGUA, 15-18.

Ríos-Touma, B., Encalada, AC., \& Prat, N. (2011). Macroinvertebrate assemblages of an Andean highaltitude tropical stream: The importance of season and flow. International Review of Hydrobiology, 96(6), 667-685.

Rivera, JJ., Pinilla, G., \& Camacho, DL. (2013). Macroinvertebrate Trophic Groups in an Andean Wetland of Colombia. Acta Biológica Colombiana, 18(2), 279-292.

Roldán, G. (2003). Bioindicación de la calidad del agua en Colombia: propuesta para el uso del método BMWP Col. (Universidad de Antioquia, Ed.). BOOK, Antioquia. Colombia: Editorial 
Universidad de Antioquia.

Roldán, G., \& Ramírez, J. (2008). Fundamentos de limnología neotropical. (F. y N. Academia Colombiana de Ciencias Exactas \& - U. C. de O. - U., Eds.) (2a ed.). Bogota, Colombia: Editorial Universidad de Antioquia.

Rosenberg, DM., \& Resh, V. (1993). Freshwater biomonitoring and benthic macroinvertebrates. In Freshwater biomonitoring and benthic macroinvertebrates (p. 488). CHAP, New York, U.S.A.: Chapman \& Hall.

Valverde, N., Caicedo, O., \& Aguirre-Ramirez, N. (2009). Análisis de calidad de agua de la quebrada La Ayurá con base en variables fisicoquímicas y macroinvertebrados acuáticos. Producción Más Limpia, 4, 44-60.

Wilhm, JL., \& Dorris, TC. (1968). Biological Parameters for Water Quality Criteria. BioScience, 18(6), 477481. 